\title{
Comparison of Human Selenoprotein P Determinants in Serum between Our Original Methods and Commercially Available Kits
}

\author{
Yoshiro Saito, ${ }^{*, a}$ Hirofumi Misu, ${ }^{b, c}$ Hiroaki Takayama, ${ }^{b, d}$ Shin-ichiro Takashima, ${ }^{d}$ Soichiro Usui, ${ }^{d}$ \\ Masayuki Takamura, ${ }^{d}$ Shuichi Kaneko, ${ }^{d}$ Toshinari Takamura, ${ }^{b}$ and Noriko Noguchi ${ }^{a}$ \\ ${ }^{a}$ Systems Life Sciences Laboratory, Department of Medical Life Systems, Faculty of Life and Medical Sciences, \\ Doshisha University; Kyoto 610-0394, Japan: ${ }^{b}$ Department of Endocrinology and Metabolism, Kanazawa University \\ Graduate School of Medical Sciences; Kanazawa, Ishikawa 920-8641, Japan: ${ }^{c}$ PRESTO, Japan Science and \\ Technology Agency; Kawaguchi, Saitama 332-0012, Japan: and ${ }^{d}$ Department of Disease Control and Homeostasis, \\ Kanazawa University Graduate School of Medical Sciences; Kanazawa, Ishikawa 920-8641, Japan. \\ Received January 22, 2018; accepted February 15, 2018
}

Selenoprotein $\mathrm{P}(\mathrm{SeP})$ is a selenium (Se)-rich extracellular protein. SeP is identified as a hepatokine, causing insulin resistance in type 2 diabetes. Thus, the measurement of $\mathrm{SeP}$ in serum has received much attention, and several enzyme-linked immunosorbent assay (ELISA) kits for SeP determination are now commercially available. In the present study, we determined the serum SeP levels by our original ELISA and sol particle homogeneous immunoassay (SPIA) methods and also by commercially available kits, and these determinants were compared. We found a kit-dependent correlation of the determinants with our methods. These results suggest that the selection of kit is critical for comparison with our previous reports and for discussing the relationship between the serum SeP levels and disease condition.

Key words selenoprotein P; monoclonal antibody; enzyme-linked immunosorbent assay (ELISA); sol particle homogeneous immunoassay (SPIA); commercially available kit

Selenoprotein P (SeP; encoded by SELENOP) is a selenium (Se)-rich extracellular glycoprotein synthesized primarily by the liver. ${ }^{1)}$ SeP contains the essential trace element Se as selenocysteine (Sec; an analog of cysteine having Se instead of sulfur), which is encoded by UGA codon in mRNA, formerly known as a stop codon. ${ }^{2}$ A stemloop RNA structure, called the Sec insertion sequence (SECIS), is essential for the incorporation of Sec during the biosynthesis of selenoproteins. The ' $\mathrm{P}$ ' in SeP denotes its presence in plasma.' SeP functions as a Se transporter to maintain levels of selenoproteins, including antioxidative enzymes such as glutathione peroxidases (GPxs). SeP has multifunctional properties such as GPx-like enzyme activity and heavy metal binding activity. ${ }^{4)}$ Thus, SeP plays a significant role in the antioxidative system in both a direct (GPx-like enzyme activity) and an indirect manner (Se transporter). We have previously reported that SeP serves as a hepatokine, promoting glucose intolerance and insulin resistance in type 2 diabetes. ${ }^{5)} \mathrm{SeP}$ is upregulated in the liver of type 2 diabetes patients, and high levels of SeP impair insulin signaling and glucose metabolism in both liver and muscle of mice. ${ }^{5)}$ We have recently reported that $\mathrm{SeP}$ is involved in exercise resistance by suppressing the levels of exercise-induced reactive oxygen species (ROS) in skeletal muscle. ${ }^{6)}$ Furthermore, it has been discovered that excess SeP decreases both pancreatic insulin levels and insulin secretion induced by high glucose. ${ }^{7)}$ Collectively, previous studies suggest that excess $\mathrm{SeP}$ is a significant therapeutic target for type 2 diabetes.

Preparation of recombinant selenoprotein for biochemical experiments is still difficult because of the complicated translational mechanism of UGA codon as Sec, particularly for $\mathrm{SeP}$, possessing $10 \mathrm{Sec}$ residues per polypeptide. ${ }^{1,2)} \mathrm{We}$ have established a method for purifying SeP protein from human plasma, ${ }^{8)}$ and purified SeP protein was used for the preparation of monoclonal antibodies (mAbs). ${ }^{9)} \mathrm{SeP}$ is composed of two domains: one Sec residue in the N-terminal region functions as the catalytic center for GPx-like enzyme activity and the other nine Sec residues in the C-terminal region functions as a Se transporter (Supplementary Fig. 1A). ${ }^{10)}$ These domains are connected by a bridge containing histidine-rich regions, each of which has the typical heparin-binding motif XBBXB ( $B$ is a basic amino acid). The digestion of full-length SeP (FL-SeP) with plasma kallikrein results in the limited proteolysis (Arg-235-Gln-236 and Arg-242-Asp-243), which generates $\mathrm{N}$-terminal (SeP-NF) and $\mathrm{C}$-terminal fragments of $\mathrm{SeP}$ (SeP-CF) (Supplementary Fig. 1A). ${ }^{10)}$ Domain structures of $\mathrm{SeP}$ are defined by using these $\mathrm{SeP}$ fragments. The rough epitope of prepared mAbs is also determined. Based on these results, we have previously developed an enzyme-linked immunosorbent assay (ELISA) specific for FL-SeP, and for both FL-SeP and SeP-NF, referred to as total SeP (Supplementary Figs. 1B, C). We further developed a sol particle homogeneous immunoassay (SPIA) for measuring the serum FL-SeP levels, which is applicable to various clinical analyzers. ${ }^{11)}$

Recently, measurement of human $\mathrm{SeP}$ has received much attention, and several ELISA kits for human SeP have become commercially available. In the present study, we compared the $\mathrm{SeP}$ determinants of our methods with those of three kinds of commercially available ELISA kits.

\section{MATERIALS AND METHODS}

Reagents Rat anti-human SeP mAbs (Clone AA3, AH5, and BD3) used in the present study were prepared as described previously. ${ }^{9)}$ Human SeP was purified from human plasma, as described previously. ${ }^{8}$ Human frozen plasma was purchased from the Japanese Red Cross Kinki Block Blood Center (No. 25J0012).

Subjects Blood samples were obtained from patients 

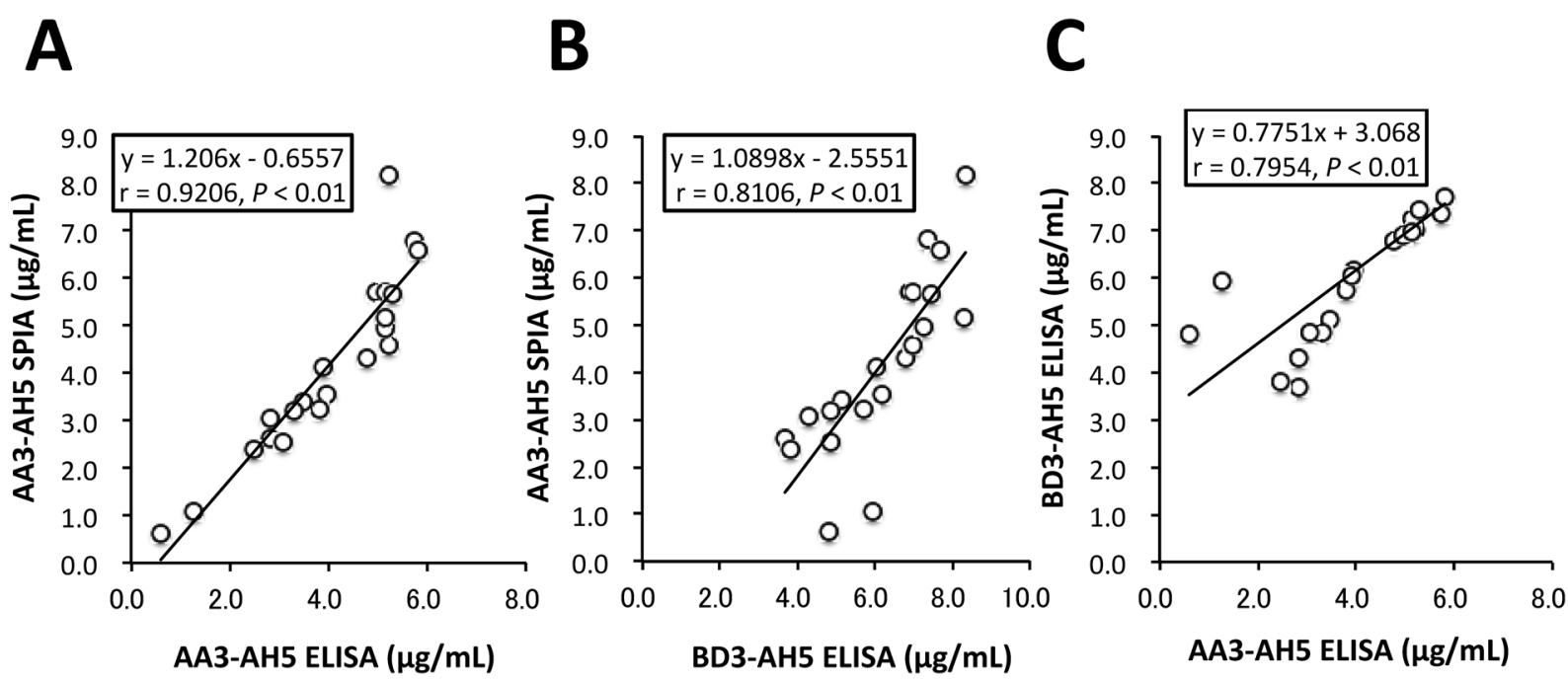

Fig. 1. Correlation between SPIA for FL-SeP and ELISA for FL-SeP and Total SeP

Serum samples $(n=21)$ were assayed by using each method, and the correlation between determinants was evaluated. The assay system used is as follows: (A) AA3-AH5 ELISA for FL-SeP $(x)$ and AA3-AH5 SPIA for FL-SeP $(y)$; (B) BD3-AH5 ELISA for total SeP $(x)$ and AA3-AH5 SPIA for FL-SeP $(y)$; and (C) AA3-AH5 ELISA for FL$\mathrm{SeP}(x)$ and BD3-AH5 ELISA for total SeP $(y)$. The solid line represents the regression line. Formula, $r$, and $p$ values are shown in each figure.
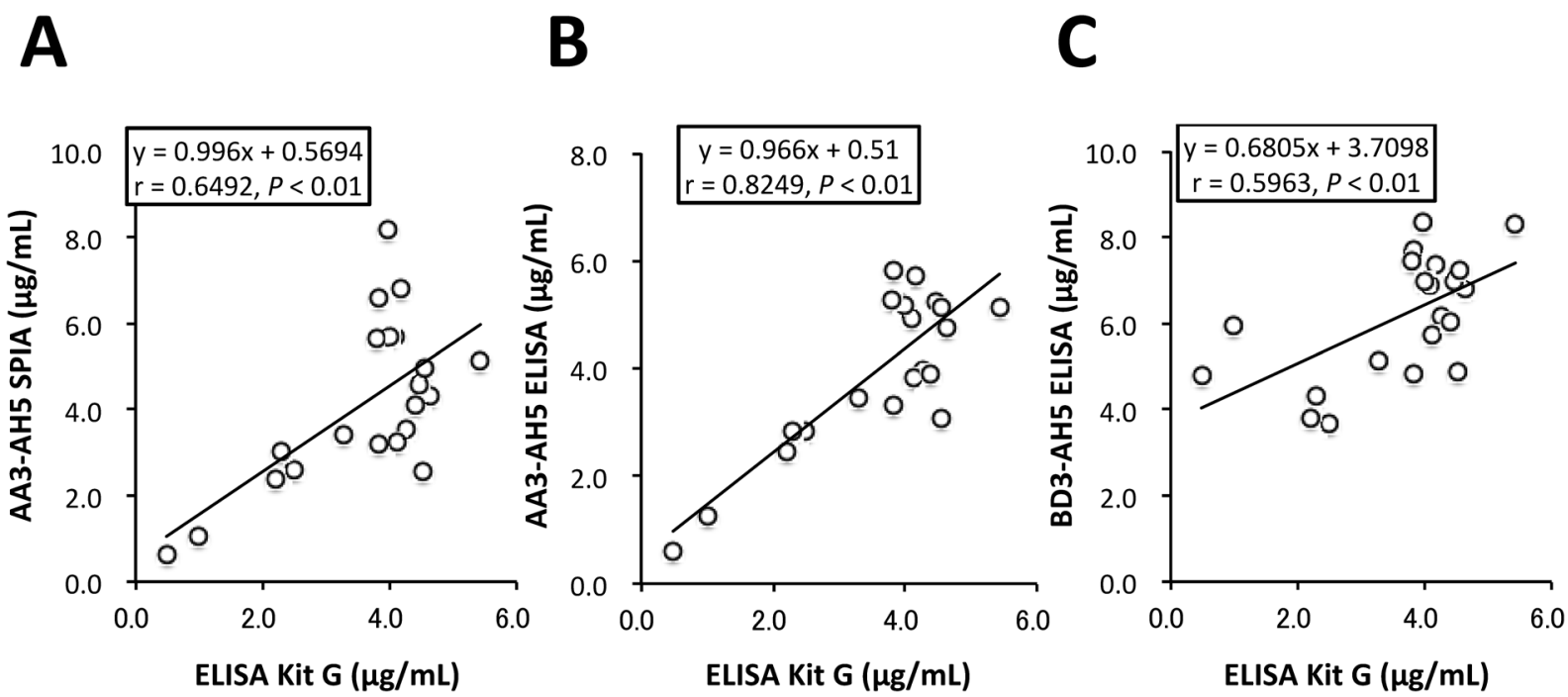

Fig. 2. Correlation of Determinants between Our Methods and Selenotest ELISA (Kit G)

(A-C) Serum samples $(n=21)$ were assayed by using each method-such as AA3-AH5 SPIA for FL-SeP, AA3-AH5 ELISA for FL-SeP, BD3-AH5 ELISA for total SeP, and Selenotest ELISA (Kit G) — and the correlation between determinants was evaluated. The solid line represents the regression line. Formula, $r$, and $p$ values are shown in each figure.

with suspected ischemic heart disease who were admitted to Kanazawa University Hospital from 2010 to 2011. Written informed consent for this study was provided from all patients. The experimental protocol was approved by the Ethical Committee of Kanazawa University (Approval No. 2152-1). The current study was conducted in accordance with the Declaration of Helsinki. A total of 21 serum samples were analyzed, and the subjects comprised eighteen males and three females (mean age \pm standard deviation, 67.2 \pm 10.0 ). Following an overnight fast, venous blood samples were obtained from each subject.

Measurement of FL-SeP and Total SeP by ELISA Measurement of FL-SeP and total SeP by ELISA was conducted, as described previously. ${ }^{9}$ ) Ninety-six-well microtiter plates were coated with $100 \mu \mathrm{L}$ of $5 \mu \mathrm{g} / \mathrm{mL}$ rat anti-human $\mathrm{SeP}$ mAb AA3 (for FL-SeP) or BD3 (for total $\mathrm{SeP}$ ) in $0.05 \mathrm{M}$ sodium bicarbonate buffer $(\mathrm{pH} 9.6)$ for $1 \mathrm{~h}$ at room temperature. The wells were washed with phosphate buffered saline (PBS) containing $0.05 \%$ Tween 20 (wash buffer) and incubated at $37^{\circ} \mathrm{C}$ with PBS containing $0.1 \%$ bovine serum albumin (BSA) (Blocking buffer) for $1 \mathrm{~h}$. After washing, $50 \mu \mathrm{L}$ of FL-SeP standard or serum sample diluted with PBS containing $0.05 \%$ Tween 20 and $0.1 \%$ BSA (PBS-Tween-BSA) was added to each well and incubated at $37^{\circ} \mathrm{C}$ for $1 \mathrm{~h}$. After washing, $50 \mu \mathrm{L}$ of horseradish peroxidase (HRP)-conjugated rat anti-human SeP mAb AH5 $(20 \mu \mathrm{g} / \mathrm{mL})$ was added and incubated at $37^{\circ} \mathrm{C}$ for $1 \mathrm{~h}$. Finally, the plates were washed, and fifty microliters of 3,3',5,5'-tetramethylbenzidine (TMB, Merck, Darmstadt, Germany) was added to each well, and the enzyme-substrate reaction was allowed to proceed for $30 \mathrm{~min}$ in the dark. The reactions were stopped by the addition of $50 \mu \mathrm{L}$ of $1 \mathrm{M}$ sulfuric acid to each well. The absorbances were read at $450 \mathrm{~nm}$ on an OPTImax plate reader (Molecular Devices, Sunnyvale, CA, U.S.A.). Wells receiving all reagents apart from serum sample 

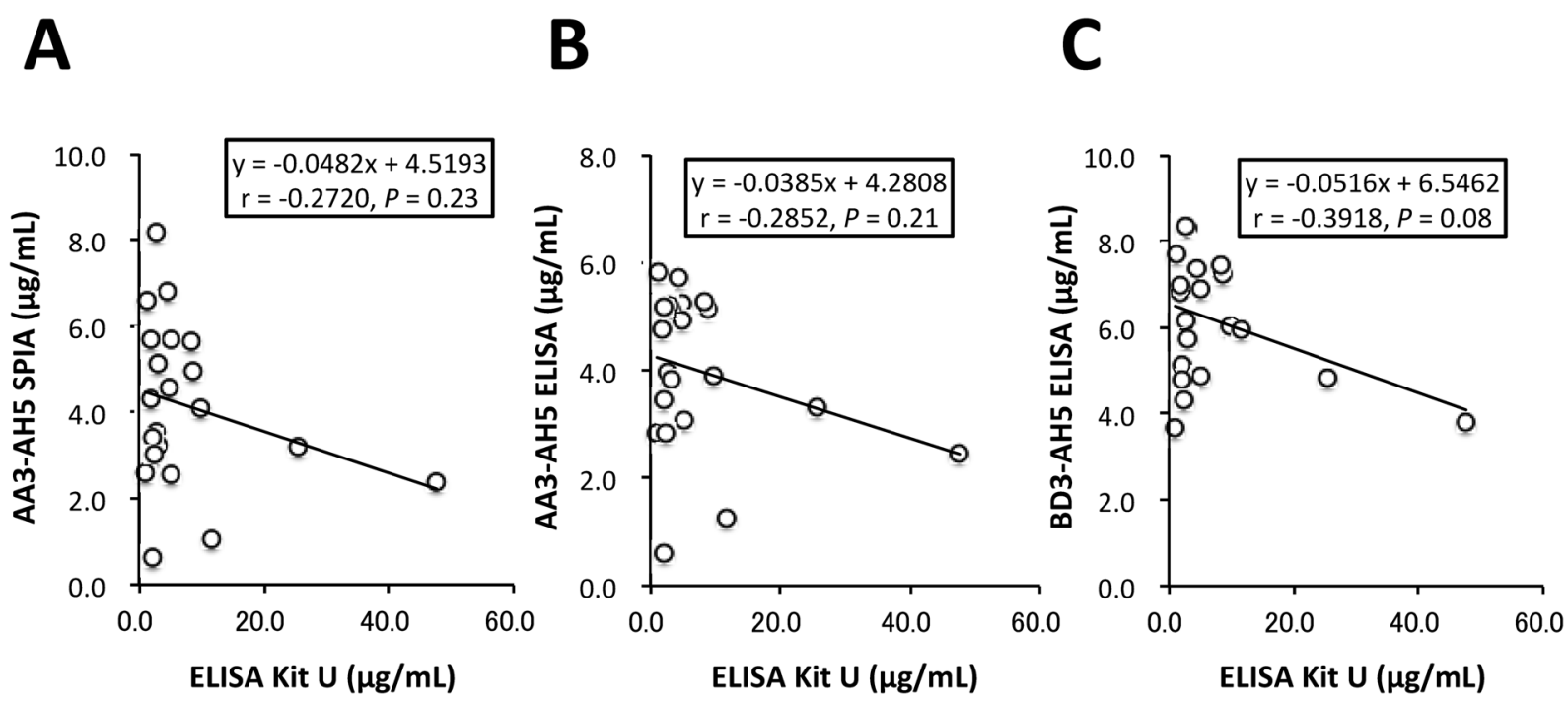

Fig. 3. Correlation of Determinants between Our Methods and ELISA Kit for Selenoprotein P1 (Kit U)

$($ A-C) Serum samples $(n=21)$ were assayed by using each method-such as AA3-AH5 SPIA for FL-SeP, AA3-AH5 ELISA for FL-SeP, BD3-AH5 ELISA for total SeP, and ELISA Kit for Selenoprotein P1 (Kit U) - and the correlation between determinants was evaluated. The solid line represents the regression line. Formula, $r$, and $p$ values are shown in each figure.
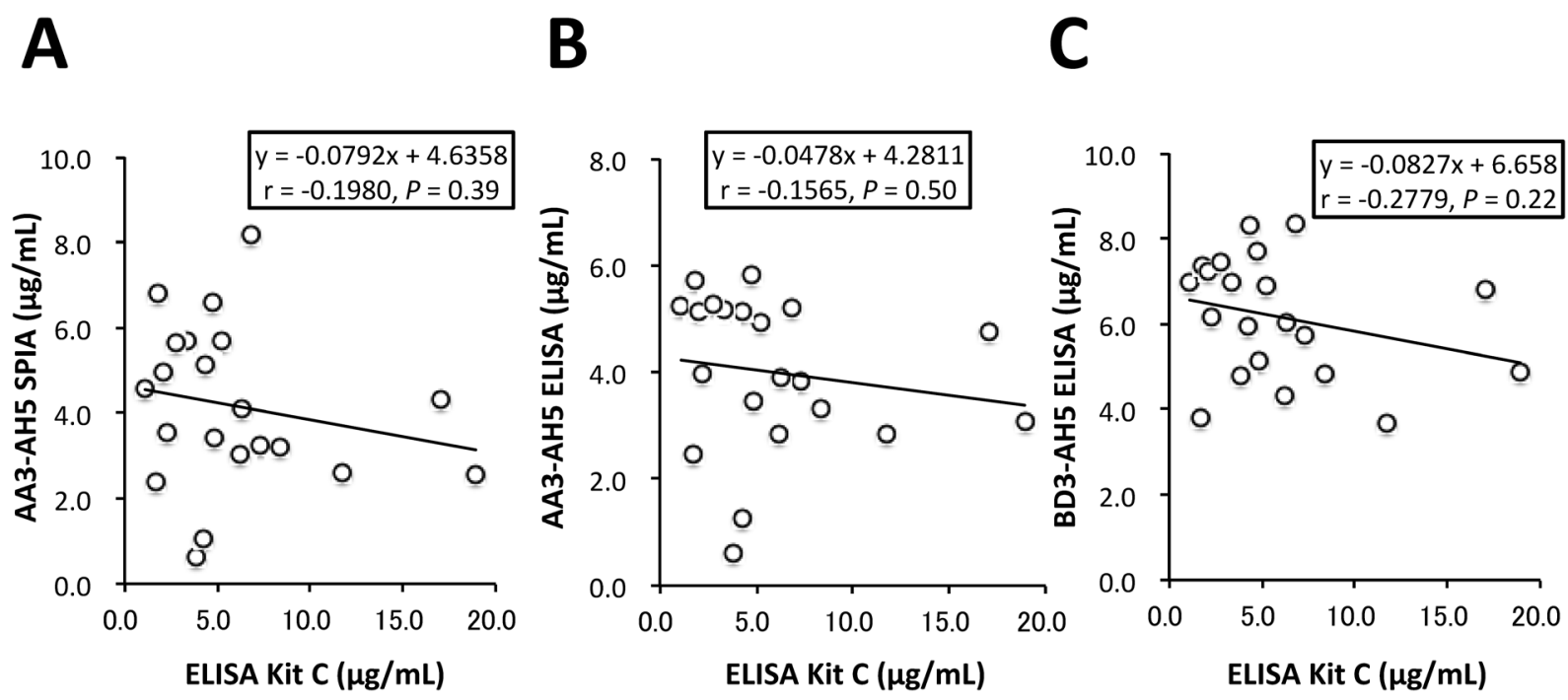

Fig. 4. Correlation of Determinants between Our Methods and Human Selenoprotein P (SEPP1) ELISA Kit (Kit C)

(A-C) Serum samples $(n=21)$ were assayed by using each method-such as AA3-AH5 SPIA for FL-SeP, AA3-AH5 ELISA for FL-SeP, BD3-AH5 ELISA for total SeP, and Human Selenoprotein P(SEPP1) ELISA kit (Kit C) — and the correlation between determinants was evaluated. The solid line represents the regression line. Formula, $r$, and $p$ values are shown in each figure.

were used as blanks.

Measurement of FL-SeP by SPIA Measurement of FLSeP by SPIA was conducted using a Model 7180 Hitachi automatic clinical analyzer (Hitachi, Tokyo, Japan), as described previously. ${ }^{11)}$ Colloidal particles coated with rat anti-human $\mathrm{SeP} \mathrm{mAb}$ were prepared as described previously. ${ }^{11)}$ An aliquot of the sample $(2 \mu \mathrm{L})$ was pipetted into a cuvette, followed by reaction buffer $(120 \mu \mathrm{L})$. The composition of reaction buffer is as follows: $0.25 \mathrm{M}$ Tris-buffer $(\mathrm{pH}$ 7.8) containing $50 \mathrm{mg} / \mathrm{mL} \mathrm{NaCl}, 2 \mathrm{mg} / \mathrm{mL}$ ethylenediaminetetetraacetic acid disodium salt (EDTA 2Na), $23 \mathrm{mg} / \mathrm{mL}$ polyethylene glycol 20000, $3.4 \mathrm{mg} / \mathrm{mL}$ gamma-butyrolactone, $3.5 \mathrm{mg} / \mathrm{mL}$ polyoxyethylene lauryl ether (Brij 35, Nacalai Tesque, Kyoto, Japan), and $2 \mathrm{mg} / \mathrm{mL}$ dodecyl(sulfonatophenoxy)benzenesulfonate (Pelex SS-H, Kao Corporation, Tokyo, Japan). After $5 \mathrm{~min}$ at $37^{\circ} \mathrm{C}$, a mixture of colloidal gold particles coated with
AH5 and AA3 $(60 \mu \mathrm{L})$ was added and mixed. The reaction between the particles and FL-SeP in the samples resulted in the formation of agglutinates and a concomitant change in the absorbance signal. After the agglutination reaction started, the absorbance values at $505 \mathrm{~nm}$ (primary wavelengths) and $660 \mathrm{~nm}$ (secondary wavelengths) were measured for $5 \mathrm{~min}$ immediately. The differences between initial absorbance and the absorbance $5 \mathrm{~min}$ after the initiation of the reaction were calculated. By using the spline program of a Model 7180 Hitachi automatic clinical analyzer, a standard curve for FL-SeP was calculated.

Measurement of SeP by Using Commercially Available Kits The following kits were used for measurement of SeP in the present study: Selenotest ELISA (InVivo BioTech Services $\mathrm{GmbH}$, Berlin, Germany), ${ }^{12)}$ ELISA Kit for Selenoprotein P1, Plasma (Cloud-Clone, Houston, U.S.A.), and Human 
Selenoprotein P (SEPP1) ELISA kit (CUSABIO, Wuhan, China). SeP content was measured according to the supplier's protocol, and the standard material of each kit was used for the determinants.

Statistical Analysis Statistical analyses were performed using Excel software and Statcel 3. Correlation coefficients were assessed using Pearson's correlation coefficient test and are shown in each figure. The regression line is also indicated. $p<0.05$ was considered to be significant.

\section{RESULTS AND DISCUSSION}

Correlation of Serum SeP Levels between Our ELISA and SPIA Method Twenty-one human serum samples with different concentrations of SeP were subjected to ELISA and SPIA for SeP using different mAbs. The rough epitope of mAbs used in the present study is as follows: AH5 and BD3, N-terminal region; AA3, C-terminal region (Supplementary Fig. 1A). ${ }^{7)}$ Therefore, the measurement system using AA3AH5 is for FL-SeP, while BD3-AH5 is for total SeP, containing both FL-SeP and SeP-NF (Supplementary Figs. 1B, C). The significant correlation between AA3-AH5 ELISA and AA3-AH5 SPIA suggests that these mAbs are working well in both systems (Fig. 1A). In addition, we found good correlation between BD3-AH5 ELISA and AA3-AH5 SPIA (Fig. 1B), and between AA3-AH5 ELISA and BD3-AH5 ELISA (Fig. 1C), suggesting small variation of SeP-NF levels in serum samples used in the present study.

Comparison between Serum SeP Determinants of Our Method and Those of Commercially Available Kits The determinants of our methods using AA3-AH5 SPIA, AA3AH5 ELISA, and BD3-AH5 ELISA were significantly correlated with those of Selenotest ELISA (Kit G) from InVivo BioTech Services GmbH Germany (Figs. 2A-C). In the case of Kit G, ELISA for SeP was developed by two kinds of antiSeP mAbs, which were prepared by using recombinant mutant SeP protein (all Sec replaced with cysteine) as immunogen. ${ }^{13)}$ Although the precise epitope of these mAbs has not been reported, the good correlation of the determinants in Kit G with those of our FL-SeP method suggests that Selenotest ELISA determines the FL-SeP.

Next, we further evaluated the correlation of determinants between our methods and ELISA Kit for Selenoprotein P1, Plasma (SEPP1) (Kit U) from Cloud-Clone U.S.A.; however, a significant correlation between these was not observed (Figs. $3 \mathrm{~A}-\mathrm{C})$. In the case of $\mathrm{Kit} \mathrm{U}$, although precise preparation of Abs used was not described in the instructions, it appears that recombinant SeP protein from Tyr 60 to Ser 299 (Sec is not included in this sequence) might be used as an immunogen. The high values of SeP, from 20 to $60 \mu \mathrm{g} / \mathrm{mL}$, in Kit U were obvious, and it is unclear whether unknown fragments of SeP are present in the human serum samples.

In the case of Human Selenoprotein P (SEPP1) ELISA kit (Kit C) from CUSABIO China, a significant correlation with our system was also not observed (Figs. 4A-C). Precise preparation of Abs used in Kit $\mathrm{C}$ was not described in the instructions, and it appears that recombinant mutant $\mathrm{SeP}$ protein (all Sec replaced with Ser) might be used as an immunogen. Similar to the determinants of Kit $\mathrm{U}$, the high values of SeP, from 10 to $20 \mu \mathrm{g} / \mathrm{mL}$, were observed. We also evaluated the correlation of determinants between Kit $\mathrm{U}$ and $\mathrm{Kit} \mathrm{C}$; how- ever, a significant correlation between these was not observed (Supplementary Fig. 2).

In the present study, we compared the determinants of serum SeP of our ELISA and SPIA methods with those of commercially available kits. We found a correlation between our determinants and kit $\mathrm{G}$, but not kit $\mathrm{U}$ or kit $\mathrm{C}$. These results suggest that the choice of kit is critical for discussing about serum levels of human SeP including the reproducibility of our previous results.

Acknowledgments This work was supported in part by KAKENHI Grant Number 25292078 and 17H03821 from the Japan Society for the Promotion of Science (JSPS) and Ministry of Education, Culture, Sports, Science and Technology (MEXT)-Supported Program for the Strategic Research Foundation at Private Universities.

Conflict of Interest The authors declare no conflict of interest.

Supplementary Materials The online version of this article contains supplementary materials.

\section{REFERENCES}

1) Burk RF, Hill KE. Selenoprotein P-expression, functions, and roles in mammals. Biochim. Biophys. Acta, 1790, 1441-1447 (2009).

2) Labunskyy VM, Hatfield DL, Gladyshev VN. Selenoproteins: molecular pathways and physiological roles. Physiol. Rev., 94, 739-777 (2014).

3) Burk RF, Hill KE. Selenoprotein P: an extracellular protein with unique physical characteristics and a role in selenium homeostasis. Annu. Rev. Nutr., 25, 215-235 (2005).

4) Saito Y, Takahashi K. Selenoprotein P. (Junqiu L, Guimin L, and Ying $\mathrm{M}$ eds.) Selenoproteins Mimics, Vol. 5, Springer-Verlag GmbH, Berlin, pp. 77-88 (2012).

5) Misu H, Takamura T, Takayama H, Hayashi H, Matsuzawa-Nagata N, Kurita S, Ishikura K, Ando H, Takeshita Y, Ota T, Sakurai M, Yamashita T, Mizukoshi E, Yamashita T, Honda M, Miyamoto K, Kubota T, Kubota N, Kadowaki T, Kim HJ, Lee IK, Minokoshi Y, Saito Y, Takahashi K, Yamada Y, Takakura N, Kaneko S. A liverderived secretory protein, selenoprotein $\mathrm{P}$, causes insulin resistance. Cell Metab., 12, 483-495 (2010).

6) Misu H, Takayama H, Saito Y, Mita Y, Kikuchi A, Ishii KA, Chikamoto K, Kanamori T, Tajima N, Lan F, Takeshita Y, Honda M, Tanaka M, Kato S, Matsuyama N, Yoshioka Y, Iwayama K, Tokuyama K, Akazawa N, Maeda S, Takekoshi K, Matsugo S, Noguchi N, Kaneko S, Takamura T. Deficiency of the hepatokine selenoprotein $\mathrm{P}$ increases responsiveness to exercise in mice through upregulation of reactive oxygen species and AMP-activated protein kinase in muscle. Nat. Med., 23, 508-516 (2017).

7) Mita Y, Nakayama K, Inari S, Nishito Y, Yoshioka Y, Sakai N, Sotani K, Nagamura T, Kuzuhara Y, Inagaki K, Iwasaki M, Misu H, Ikegawa M, Takamura T, Noguchi N, Saito Y. Selenoprotein P-neutralizing antibodies improve insulin secretion and glucose sensitivity in type 2 diabetes mouse models. Nat. Commun., 8, 1658 (2017).

8) Saito Y, Hayashi T, Tanaka A, Watanabe Y, Suzuki M, Saito E, Takahashi K. Selenoprotein $\mathrm{P}$ in human plasma as an extracellular phospholipid hydroperoxide glutathione peroxidase. Isolation and enzymatic characterization of human selenoprotein P. J. Biol. Chem., 274, 2866-2871 (1999).

9) Saito Y, Watanabe Y, Saito E, Honjoh T, Takahashi K. Production and application of monoclonal antibodies to human selenoprotein $\mathrm{P}$. 
J. Health Sci., 47, 346-352 (2001).

10) Saito Y, Sato N, Hirashima M, Takebe G, Nagasawa S, Takahashi $\mathrm{K}$. Domain structure of bi-functional selenoprotein P. Biochem. J., 381, 841-846 (2004).

11) Tanaka M, Saito Y, Misu H, Kato S, Kita Y, Takeshita Y, Kanamori T, Nagano T, Nakagen M, Urabe T, Takamura T, Kaneko S, Takahashi K, Matsuyama N. Development of a sol particle homogeneous immunoassay for measuring full-length selenoprotein $\mathrm{P}$ in human serum. J. Clin. Lab. Anal., 30, 114-122 (2016).
12) Hybsier S, Schulz T, Wu Z, Demuth I, Minich WB, Renko K, Rijntjes E, Köhrle J, Strasburger CJ, Steinhagen-Thiessen E, Schomburg L. Sex-specific and inter-individual differences in biomarkers of selenium status identified by a calibrated ELISA for selenoprotein P. Redox Biol., 11, 403-414 (2017).

13) Hybsier S, Wu Z, Schulz T, Strasburger CJ, Köhrle J, Minich WB, Schomburg L. Establishment and characterization of a new ELISA for selenoprotein P. Perspect. Sci., 3, 23-24 (2015). 